Trans

continentales
Transcontinentales

Sociétés, idéologies, système mondial

$4 \mid 2007$

Amérique latine

\title{
L'enseignement supérieur dans le monde arabe
}

Mounir BASHSHUR, Youssef COURBAGE et Boutros LABAKI (dir.),

L'enseignement supérieur dans le monde arabe, une question de niveau, Beyrouth, IFPO, 2006, 214 p.

\section{Mustapha Haddab}

\section{OpenEdition}

\section{Journals}

Édition électronique

URL : http://journals.openedition.org/transcontinentales/1000

DOI : 10.4000/transcontinentales. 1000

ISBN : 978-2-7351-1565-5

ISSN : 1775-397X

Éditeur

Editions de la maison des sciences de l'homme

\section{Édition imprimée}

Date de publication : 1 juin 2007

Pagination : 169-171

ISBN : 978-2-200-92396-9

ISSN : 1950-1684

Référence électronique

Mustapha Haddab, "L'enseignement supérieur dans le monde arabe », Transcontinentales [En ligne], 4 | 2007, document 10, mis en ligne le 04 mai 2011, consulté le 25 septembre 2020. URL : http://

journals.openedition.org/transcontinentales/1000; DOI : https://doi.org/10.4000/transcontinentales. 1000 
L'enseignement supérieur dans le monde arabe

\section{Mounir BASHSHUR, Youssef COURBAGE et Boutros LABAKI (dir.), L'enseignement supérieur dans le monde arabe, une question de niveau, Beyrouth, IFPO, 2006, 214 p.}

Une des qualités de cet ouvrage est d'être dans une certaine mesure en rupture avec la tendance de nombreuses études consacrées aux systèmes nationaux d'enseignement supérieur, tendance consistant en particulier à proposer des descriptions essentiellement quantitatives de ces systèmes et de leur évolution, et à établir, en usant d'indicateurs classiques comme le taux de réussite, d'encadrement, de féminisation, etc., des bilans qui font souvent l'objet d'interprétations peu rigoureuses, parfois inspirées par des visées politiques, idéologiques ou même corporatistes.

En choisissant de s'interroger sur les conditions d'une évaluation qualitative des systèmes d'enseignement supérieur de plusieurs pays arabes, les participants de l'atelier international sur «le niveau de l'enseignement supérieur dans le monde arabe», tenu à Beyrouth les 17 et 18 mars 2005 à l'initiative de l'Institut français du Proche-Orient en partenariat avec la Fondation Ford pour le MoyenOrient et l'Afrique du Nord - atelier à l'origine de cet ouvrage -, adoptent une démarche qui nécessite un examen plus fin et plus critique des statistiques disponibles et une bonne connaissance de la dimension socio-historique du fonctionnement des institutions universitaires.

Comme le font en outre bien apparaitre Youssef Courbage dans sa présentation, et Mounir Bashshur dans la synthèse qui clôt cet ouvrage, la réunion dans ce livre d'études portant sur six systèmes univer- sitaires nationaux (libanais, algérien, palestinien, égyptien, syrien et marocain) fournit la matière à des comparaisons éclairantes entre les caractéristiques de ces systèmes, comparaisons conduisant à prendre une mesure plus juste aussi bien des points forts que des points faibles des institutions universitaires des pays arabes concernés, en incitant à plus de rigueur dans la formulation d'hypothèses explicatives des phénomènes observés.

La lecture des différentes contributions à cet ouvrage permet par exemple de vérifier que la question de la gestion des effets de la démocratisation de l'accès aux études supérieures se pose d'une manière comparable dans tous les pays arabes concernés. Ce qui, entre autres aspects, donne à penser que la forte tendance à l'augmentation des effectifs d'étudiants résulte de facteurs sociaux profonds et pas seulement du «volontarisme» des gouvernants.

Ces derniers sont partout confrontés à la dévalorisation sociale de beaucoup de diplômes universitaires, due en partie à une croissance des effectifs d'étudiants que n'accompagne pas une croissance économique créatrice d'un nombre significatif $\mathrm{d}^{\prime}$ empois qualifiés. Le nombre d'étudiants a par exemple quintuplé en Égypte entre 1976 et 2002; il a quadruplé en Algérie entre 1995 et 2005; il a été multiplié par quatre en Palestine durant les deux dernières décennies, etc.

Dans tous les pays arabes étudiés, une sorte de cercle vicieux s'instaure, devant lequel les pouvoirs publics paraissent assez démunis : l'augmentation des effectifs d'élèves et d'étudiants entraîne une importante dévalorisation des diplômes, processus qui entraîne à son tour une tendance, chez ces élèves et étudiants, à 
prolonger la durée de leurs cursus dans le but d'atteindre à des niveaux d'études qui pourraient, espèrent-ils, les protéger du chômage.

La surpopulation qui en résulte dans les institutions universitaires tend à rendre très difficile l'application de mesures destinées à améliorer la qualité des formations données aux étudiants; ces mesures sont, pour la majorité des universités des pays arabes étudiés dans cet ouvrage, d'autant plus timides et peu efficaces qu'elles ne peuvent le plus souvent être appuyées sur des allocations budgétaires suffisantes. Les salaires des enseignants demeurent le plus souvent insuffisants, ce qui, entre autres conséquences, incite ces derniers à diversifier leurs sources de revenus au détriment de l'exercice de leur métier et de leur assiduité à conduire des recherches; les bibliothèques, les laboratoires, les conditions de vie de la majorité des étudiants restent le plus souvent médiocres. L'augmentation des budgets de fonctionnement des universités publiques conduit souvent à la réduction des sommes consacrées à la recherche scientifique. Au Maroc, par exemple, le budget de la recherche scientifique ne représente qu'un cinquième de celui de l'enseignement supérieur (M. Souali, p. 195). "[... L]'expansion [des effectifs d'étudiants] est en corrélation avec une diminution sensible de la moyenne des dépenses consacrées à chaque étudiant», lit-on dans le texte consacré par M. Zaytoun à la situation de l'enseignement supérieur en Égypte (p. 116, traduction M. Haddab).

Autre dilemme auquel se trouve confrontée la gestion des systèmes universitaires des pays arabes: l'amélioration de la qualité et du niveau de certaines formations ou seulement leur préservation (ce qui est souvent le cas pour, par exemple, les études médicales, les sciences de l'ingénieur et l'informatique) augmentent la probabilité de voir les diplômés les mieux formés émigrer vers d'autres cieux; autant de bons cadres et professeurs potentiels dont les institutions du pays, et particulièrement les universités, doivent se passer.

Parmi les traits communs aux systèmes d'enseignement supérieur analysés dans cet ouvrage, et que le contenu de ce dernier permet de percevoir avec netteté, on relève la tendance des étudiants à suivre majoritairement des études de lettres ou de gestion, plutôt que de s'orienter vers les sciences exactes ou les sciences de l'ingénieur. Au Maroc, en 2004, 75 \% des étudiants suivent des études de lettres, ou de droit et d'économie (p. 172). La proportion d'étudiants inscrits dans les filières des sciences fondamentales a eu, dans ce même pays, tendance à baisser : elle est passée de $43,7 \%$ en 1975 , à 22,5 \% en 2001 (p. 127). Au Liban, $48 \%$ des étudiants appartiennent en 2004 aux filières de sciences humaines, de droit et de sciences politiques et administratives (M. Bashshur, p. 12).

Ces tendances paraissent avoir, d'un pays à l'autre, des causes assez semblables telles que la croissance rapide du nombre d'étudiants, les débouchés réduits des études scientifiques et technologiques dans des pays encore peu industrialisés, et le caractère coûteux des équipements nécessaires aux filières scientifiques et technologiques.

Les facteurs auxquels sont imputées les insuffisances qualitatives des systèmes d'enseignement supérieur considérés ici ne paraissent ainsi pas très différents, d'un pays à l'autre. La coexistence de plusieurs langues d'enseignement s'accompagne à des degrés divers de difficultés d'ordre 
pédagogique, qui apparaissent aussi dans les rapports des diplômés au marché du travail, dans tous les pays étudiés dans cet ouvrage. "La question des langues s'est imposée au fil des années comme un problème central des facteurs qui altèrent la qualité de l'enseignement supérieur...» note l'auteur de l'étude consacrée au Maroc (p. 190). Non moindre est l'acuité avec laquelle ces questions persistent à se poser en Algérie.

Par les données quantitatives et qualitatives que cet ouvrage fournit, et le dépassement des cloisonnements entre pays qu'il rend possible, et aussi parce que les problèmes méthodologiques que pose une évaluation des aspects qualitatifs et pas seulement quantitatifs de l'évolution des systèmes universitaires ne sont pas éludés, ce livre constitue une base de comparaison fort utile, et suggère de nombreuses pistes et hypothèses de recherche.

Mustapha Haddab Professeur à l'université d'Alger

\section{Sexualité et procréation face au Sida}

\section{Annabel DeSGREES DU LOU \\ et Benoît Ferry (éds), \\ Sexualité et procréation confrontées \\ au Sida dans les pays du Sud, Paris, 2006, CEPED-IRD, $273 \mathrm{p}$.}

Cet ouvrage collectif pluridisciplinaire est l'aboutissement d'un colloque ayant rassemblé en 2004 des chercheurs de plusieurs continents (principalement Asie et Afrique) qui s'intéressent aux liens entre l'épidémie du Sida et les comportements sexuels et reproductifs. Partant du principe que les campagnes de prévention contre le VIH modifient l'ensemble de la sphère sexualité-contraception-procréation, les auteurs considèrent que tout le domaine de la santé de la reproduction est ainsi perturbé. Outre la diversité des questionnements mis en évidence par les auteurs aux approches très variables (de la micro-anthropologie qualitative à la socio-épidémiologie quantitative), un des atouts scientifiques du livre réside dans la richesse de son champ d'investigation. Bien que sur une problématique relativement circonscrite, l'éventail des questions abordant la sexualité et la procréation en temps de Sida dépasse largement le point de vue médical et fait apparaître la nécessité de s'intéresser à toute une pléthore de données sociales conditionnant le déroulement des programmes de réduction de la transmission mère-enfant (PMTE). À rappeler que le dépistage est présenté comme un point d'articulation fort entre la prévention et les soins à venir.

Si plusieurs contributions tentent avec plus ou moins de succès de synthétiser la somme des connaissances acquises, la contraception face au VIH par exemple, l'ouvrage montre que, quels que soient les angles d'approche, aucune "solution" ou «ligne de conduite» n'est décemment envisageable à partir de recommandations ayant valeur universelle contrairement à ce que peuvent fréquemment évoquer les organisations internationales impliquées dans la gestion de la pandémie du Sida. Une clé qui ouvre toutes les portes n'est pas une bonne clé, et peut-être est-ce une des leçons majeures de ce livre de nous montrer à maintes reprises que tout appui en matière de santé ne peut s'élaborer sans composer patiemment avec l'économie nationale, les politiques locales et les dynamiques socioculturelles aussi bien que familiales qui conditionnent toute intervention et font sens dans un contexte précis.

Plus d'une quinzaine de contributions sont distribuées en trois parties. La 